This is an electronic reprint of the original article. This reprint may differ from the original in pagination and typographic detail.

Author(s): Partanen, Jari; Johansen, U.; Sarén, Jan; Tuunanen, Juha; Uusitalo, Juha

Title: $\quad$ High voltage conditioning of the electrostatic deflector of MARA

Year: $\quad 2016$

Version:

Please cite the original version:

Partanen, J., Johansen, U., Sarén, J., Tuunanen, J., \& Uusitalo, J. (2016). High voltage conditioning of the electrostatic deflector of MARA. Nuclear Instruments and Methods in Physics Research Section B: Beam Interactions with Materials and Atoms, 376, 353-356. https://doi.org/10.1016/j.nimb.2015.11.025

All material supplied via JYX is protected by copyright and other intellectual property rights, and duplication or sale of all or part of any of the repository collections is not permitted, except that material may be duplicated by you for your research use or educational purposes in electronic or print form. You must obtain permission for any other use. Electronic or print copies may not be offered, whether for sale or otherwise to anyone who is not an authorised user. 


\title{
High voltage conditioning of the electrostatic deflector of MARA
}

\author{
J. Partanen ${ }^{\mathrm{a}, *}$, U. Johansen $^{\mathrm{b}}$, J. Sarén ${ }^{\mathrm{a}}$, J. Tuunanen ${ }^{\mathrm{a}}$, J. Uusitalo ${ }^{\mathrm{a}}$ \\ ${ }^{a}$ University of Jyväskylä, Department of Physics, FI-40014 Jyvskylä, Finland \\ ${ }^{b}$ Danfysik A/S, Gregersensvej 8, DK-2630 Taastrup, Denmark
}

\begin{abstract}
MARA is a new recoil mass separator in the Accelerator Laboratory of University of Jyväskylä (JYFL-ACCLAB) with a mass resolving power of 250 and an ion-optical configuration of $Q Q Q D_{\mathrm{E}} \mathrm{D}_{\mathrm{M}}$. In this paper the construction, control and conditioning of its electrostatic deflector are described. The deflector was designed for voltages up to $500 \mathrm{kV}$ accross the gap, corresponding to a $3.6 \mathrm{MV} / \mathrm{m}$ field, to accomodate fusion reactions with inverse kinematics. Titanium electrodes with a beam dump opening in the anode are used. The conditioning procedure, which has been used repeatedly to take the deflector to $450 \mathrm{kV}$, is described, along with the safety systems and precautions that are in place.
\end{abstract}

Table 1. Simulated properties of MARA

\begin{tabular}{cc}
\hline Optical length & $6.85 \mathrm{~m}$ \\
Configuration & $\mathrm{QQQD}_{\mathrm{E}} \mathrm{D}_{\mathrm{M}}$ \\
Electric rigidity (max) & $14 \mathrm{MV}$ \\
Magnetic rigidity (max) & $1.0 \mathrm{Tm}$ \\
Angular acceptance (horiz., vert.) & $45 \mathrm{mrad}, 55 \mathrm{mrad}$ \\
Energy acceptance & $-15-+20 \%$ \\
m/q-dispersion & $8 \mathrm{~mm} / \%$ \\
First order resolving power & 250 \\
\hline
\end{tabular}

\section{The Mass Analysing Recoil Apparatus}

MARA is a compact vacuum mode recoil separator awaiting commissioning at JYFL-ACCLAB. It complements the existing gas-filled recoil separator RITU [1]. This is the case particularly in the lower mass region near ${ }^{100} \mathrm{Sn}$, where symmetric reactions or inverse kinematics are required for fusion-evaporation with stable beams and the multitude of open evaporation channels calls for physical separation of the different reaction products. Ion-optically MARA consists of a magnetic quadrupole triplet followed by an electrostatic deflector and a magnetic dipole with a quadrupole component, as depicted in figure 1. MARA is designed to have adequate angular, energy and mass acceptance, a mass resolving power of 250 and physical separation of species with different $\mathrm{m} / \mathrm{q}$ [2]. The singledeflector construction reduces the number of optical elements and optical length of the separator compared to a symmetric solution. Key properties of the separator in a nominal configuration are listed in table 1.

\footnotetext{
${ }^{*}$ Corresponding author

Email address: japapepa@jyu.fi (J. Partanen)
}

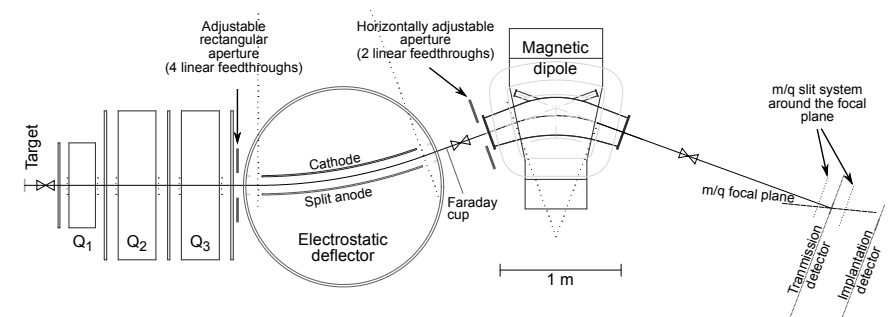

Figure 1. Layout of MARA recoil mass separator. Figure from J. Sarén's doctoral thesis.

Key properties of the deflector are listed in table 2. The deflector has a split anode with a $15 \mathrm{~mm}$ opening extending from $600 \mathrm{~mm}$ from the beginning to $137 \mathrm{~mm}$ from the end of the plate. The primary beam is dumped through this opening into a position sensitive beam dump, which will allow online monitoring of the beam intensity and focus. The opening is surrounded with a shim to minimise the effect it has on the field. Typically ions travel through only the middle $10 \mathrm{~cm}$ of the total electrode height. This oversizing allows for a simple electrode geometry while ensuring uniformity of the field in the central region. Grounded shunts are placed at the deflector entrance and exit to clamp fringing fields. The deflector is housed in a cylindrical stainless steel vacuum chamber. The vacuum volume of $8 \mathrm{~m}^{3}$ is pumped with a $S=3000 \mathrm{l} / \mathrm{s}$ cryopump, with a $S=16001 / \mathrm{s}$ turbomolecular pump on standby for cryopump regeneration. A vacuum level of $10^{-9} \mathrm{mbar}$ is reached with the deflector volume isolated. When the separator is operational and MWPC gas flow is on at the focal plane the deflector chamber pressure rises to $10^{-7} \mathrm{mbar}$. 
Table 2. Properties of the electrostatic deflector

\begin{tabular}{cc}
\hline Manufacturer & Danfysik A/S \\
Electrode material & titanium \\
Design voltage (max) & $500 \mathrm{kV}$ \\
Electrode distance & $14 \mathrm{~cm}$ \\
Bending radius & $4.0 \mathrm{~m}$ \\
Bending angle & $20^{\circ}$ \\
Beam dump opening & $15 \mathrm{~mm}$ \\
Electrode height & $46 \mathrm{~cm}$ \\
\hline
\end{tabular}

\section{Electrode design and material choices}

Electric strength is the maximum stable electric field achievable with a given pair of electrodes in a given composition and pressure of residual gas. It defines the highest electric rigidity achievable with a deflector. Sufficient electric strength is therefore paramount in ensuring that design criteria of the separator are met.

Several relatively recent electrostatic deflectors feature titanium electrodes, and that is the material of choice for MARA as well. The electric strength of titanium electrodes is very good, although inferior to an oxidised aluminium cathode and stainless steel anode, and highly stable [3]. The material is also notoriously hard, a valuable property when tight-fitting alignment tools have to be used. Furthermore, the deflector manufacturer already had previous experience in using titanium for electrodes of electrostatic devices. This last point is of great importance, when one bears in mind that machining of the electrodes should be kept to a minimum after they have been heat treated to avoid building up mechanical stress in their structure.

Sharp edges were avoided in the design of the surfaces exposed to high voltage, i.e. the electrodes themselves and all grounded surfaces close to the electrodes. The average surface roughness of the electrodes was specified as $R_{\mathrm{a}}=0.25 \mu \mathrm{m}$, but the achieved value has not been measured. Polishing of the electrodes was done without using grinding pastes.

\section{High voltage supply}

The surfaces of new electrodes are rife with impurities and microprotrusions, which serve as electron emission sites. In order to reach the high electric strength required, the electrodes have to go through a conditioning process to deal with these irregularities. The simplest and therefore most common form of conditioning is current conditioning, where electron emission sites are blunted due to thermal effects. Another popular technique is spark conditioning, where electric strength is gained by allowing the gap to spark repeatedly until the surface inhomogeneities have been removed by arc erosion [4]. Both these conditioning procsses are at play during the deflector's conditioning, although current conditioning is favoured as the faster-progressing alternative. Electrostatic devices are customarily conditioned to $10-15 \%$ above the desired stable voltage and this practice is followed also with MARA. Reconditioning is typically required at least on reapplication of high voltage, but this process is a lot faster than the first conditioning as long as the deflector has been kept in high vacuum for the entire time.

Two FuG HCH 250000 high voltage supplies are used to generate the positive and negative high voltage for the deflector that is fed to the electrodes via ceramic feedthroughs. The supplies are capable of producing a voltage of $250 \mathrm{kV}$ each and can source continuous output currents of $1 \mathrm{~mA}$. The current capacity is excessive for the application. Voltage reproducibility of the supplies is $2 \cdot 10^{-3}$. Peak current is limited only by the $500 \mathrm{k} \Omega$ series resistance on the outputs of the supplies and has been observed to go over the measurement scale of $1 \mathrm{~mA}$. Time constant of the RCcircuit formed by the supply's output capacitance and the aforementioned resistance is $500 \mu \mathrm{s}$, which is over four orders of magnitude longer than the $10 \mathrm{~ns}$ deemed safe in literature [4]. Because the high voltage is generated outside the vacuum enclosure, cable capacitance has to be taken into account as well. Specific capacitance of a typical $250 \mathrm{kV}$ rated cable is around $100 \mathrm{pF} / \mathrm{m}$ Thus, three meters of such cable stores $6 \mathrm{~J}$ of energy and $0.06 \mathrm{mC}$ of charge when biased to $200 \mathrm{kV}$. The electrode plates form a $40 \mathrm{pF}$ capacitor and the total energy and charge stored in the cables and the electrode gap are thus $15 \mathrm{~J}$ and $0.12 \mathrm{mC}$, respectively. In a study on the conditioning of a $\mathrm{CuCr}$ electrode in ultra-high vacuum [5] it was found that no electrode damage sufficient to limit the achievable electric strength occurred when charge per breakdown didn't exceed $1.2 \mathrm{mC}$. One should also note that the electric field required for the MARA deflector is nearly two orders of magnitude smaller than the fields applied in [5]. However, a more strict safe discharge energy limit of $10 \mathrm{~J}$ can also be found in literature [4].

\section{Control system}

An automated control system for the HV supplies has been built to ensure smooth, safe and reliable operation of the electric dipole. An industrial programmable logic controller from WAGO System 750 family was chosen as the control system platform for its mobility and robustness as well as ease of programming and integration to existing systems. Analogue signalling is used to transmit voltage and current values. Set values are transmitted to the supply as $0-20 \mathrm{~mA}$ current signals to better ensure signal integrity while $0-10 \mathrm{~V}$ voltage signals are used for read values. The DA converters have a 12 bit resolution, which translates to a resolution of $61 \mathrm{~V}$ for set voltage and $0.24 \mu \mathrm{A}$ for current limit, and a conversion time of $10 \mathrm{~ms}$. The AD converter used to read the measured HV has 15 bit $(8 \mathrm{~V})$ resolution and a $80 \mathrm{~ms}$ conversion time. Leakage currents are read in with a 12 bit $\mathrm{AD}$ converter to make 
use of its $4 \mathrm{~ms}$ conversion time. The system is unable to respond to overcurrent conditions lasting less than $30 \mathrm{~ms}$.

Voltage is ramped towards the goal voltage at a userset rate. The control software allows the user to set four different leakage current limits. Ramping up is stopped upon reaching stop limit $I_{\mathrm{S}}$. If lowering limit $I_{\mathrm{L}}$ is reached, the voltage is ramped down until current is once again below the limit. On discharge limit $I_{\mathrm{D}}$ the control of the affected supply goes into discharge mode, where voltage is dropped by a percentage. Another drop is made if the discharge condition persists longer than a given time interval. Lastly, a hardware limit $I_{\mathrm{H}}$ sets an absolute limit for the long term current output of the supply. This is also the limit with the shortest response time. This fourlimit approach allows for different conditioning schemes. If the discharge limit is set greater than the hard limit, the conditioning logic resembles the one used with SHIP condensers [6]. A system with lowering and discharge limit is used with CARP at RCNP [7].

A two-level vacuum interlock is incorporated into the HV control: at $5 \cdot 10^{-6}$ mbar both supplies are set into discharge mode to bring the voltage down and the deflector vacuum is isolated. A second interlock set at $5 \cdot 10^{-5} \mathrm{mbar}$ lowers the voltages abruptly to zero and doesn't reset automatically. The vacuum level measured from the deflector chamber has to remain above the limit for a second to activate the interlock. This is to account for the sharp peaks in the Penning gauge vacuum reading caused by discharges during conditioning.

\section{Conditioning of the deflector}

The deflector was conditioned at the factory using the setup described in the previous section. The voltage, leakage current, radiation level two meters from the deflector exit and vacuum level were logged. Figure 2 shows typical behaviour of the deflector vacuum and radiation level during conditioning. A discharge is typically accompanied by an increase in radiation level due to bremsstrahlung. Radiation levels as high as $500 \mu \mathrm{Sv} / \mathrm{h}$ were recorded by the Kata DGM-1500 Geiger counter used. The measures taken to ensure radiation safety can accommodate a bremsstrahlung level of $1000 \mathrm{mSv} / \mathrm{h}$ and consist of $6 \mathrm{~mm}$ thick lead shielding around the deflector, the concrete wall of the experimental cave, which is $500 \mathrm{~mm}$ thick at the thinnest point, as well as restricted access to the cave during conditioning. The lead shielding is designed to cut the radiation level down by a factor of 30 with $500 \mathrm{kV}$ accross the gap. Discharges aside, radiation level during operation averaged to $0.5 \mu \mathrm{Sv} / \mathrm{h}$. Bremsstrahlung spikes exceeding $3 \mu \mathrm{Sv} / \mathrm{h}$ could be seen at 3-10 min intervals with each deflector electrode conditioned to and kept at $205 \mathrm{kV}$, demonstrating the deconditioning effect and the need to condition the deflector to a higher voltage than the one it is going to be operated at. Events in which the leakage current exceeded $16 \mu \mathrm{A}$ were

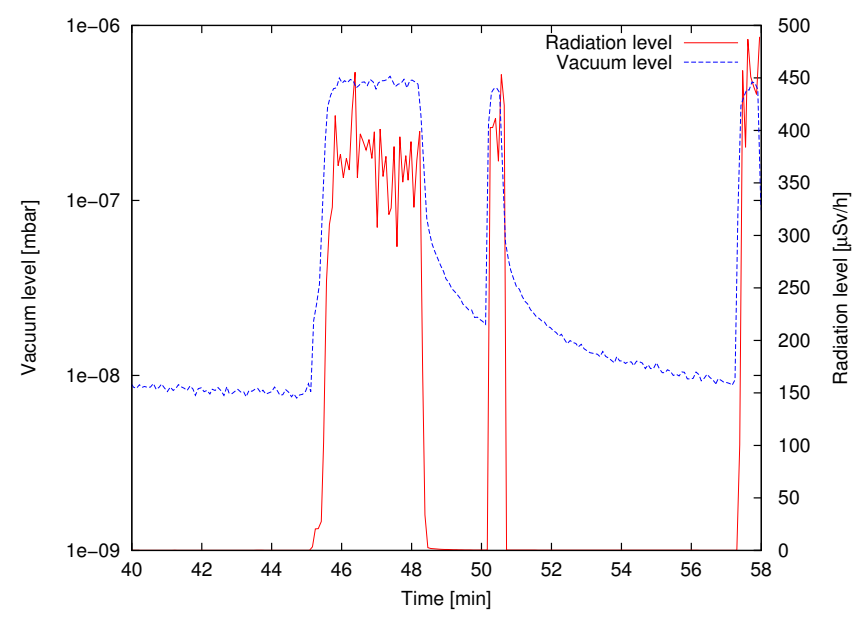

Figure 2. Vacuum and radiation levels during factory conditioning of the deflector. Both levels rise when leakage current is drawn. Data are from the same time period as the data in figure 3.

seen roughly once every two hours during the stable period. There are plans to use a Compton suppressed germanium detector array for gamma rays at MARA target position. The Compton suppression should protect against polluting the germanium spectra with bremsstrahlung. In figure 2 one also sees spikes well over an order of magnitude high in the vacuum level during discharges.

Conditioning of the deflector is done by first conditioning the electrodes individually with respect to ground and then with respect to each other. A similar procedure is used in FMA [8]. It was thought prudent to do the factory conditioning in steps of a few kilovolts, even though the control system is capable of autonomous operation. Different conditioning parameters were experimented with during the factory conditioning. For the early phases of conditioning, the current limits $I_{\mathrm{S}}=150 \mu \mathrm{A}, I_{\mathrm{L}}=$ $150 \mu \mathrm{A}, I_{\mathrm{D}}=250 \mu \mathrm{A}$ and $I_{\mathrm{H}}=250 \mu \mathrm{A}$ seemed to work well with a ramp rate of $10 \mathrm{kV} / \mathrm{s}$ and a discharge drop of $10 \%$. The difference between $I_{\mathrm{S}}, I_{\mathrm{L}}$ and $I_{\mathrm{H}}$ had to be increased and the ramp rate and discharge drop lowered to $1 \mathrm{kV} / \mathrm{s}$ and $2 \%$, respectively, to get through a bottleneck, which seemed to form at an inter-electrode voltage of $410 \mathrm{kV}$. A stable inter-electrode voltage of $440 \mathrm{kV}$ was reached in 4 hours from an unbiased state during the factory acceptance test. However, the deflector had been at a voltage of $410 \mathrm{kV}$ for several days before the test.

$\mathrm{HV}$ and leakage current values from factory conditioning are plotted in figure 3, where long-lasting elevated levels in leakage currents ("burning") can be seen on both electrodes. Such inter-electrode discharges are seen most often in latter stages of conditioning when both electrodes are biased. Current from a single electrode to ground is more prevalent when conditioning individual electrodes. The electrodes were inspected upon dismantling the deflector for transportation to Jyväskylä. Brownish marks 


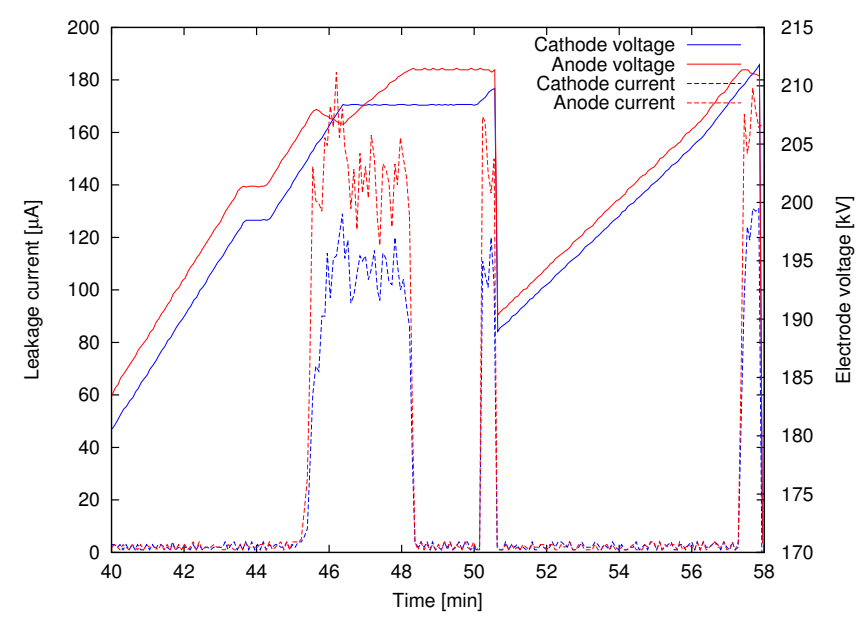

Figure 3. High voltage and leakage currents of the deflector during factory conditioning. Operation of the conditioning logic, i.e. gradual and abrupt lowering of voltages upon reaching leakage current set-points, can be seen. The current spikes triggering the abrupt drops aren't shown in the figure.

on the cathode traced the shim around the anode opening, indicating that the gap normally sparked at the shim.

The deflector electrode assembly was stored on the floor in the experimental hall, covered with a tarpaulin, for several months while the beamline was aligned. The assembly and all easily reachable grounded surfaces of the vacuum chamber were thoroughly scrubbed with isopropanol before reassembly. Conditioning parameters were optimised further during reconditioning. As a first test voltages were brought up symmetrically. First sparks were seen before $140 \mathrm{kV}$ accross the gap. Cathode was left at $-70 \mathrm{kV}$ and conditioning of the anode was commenced.

A clear difference in the behaviour of the deflector was seen compared to the factory conditioing. Sparking, i.e. leakage currents going over the measurement range, was a lot more prevalent and decreasing the discharge limit failed to reduce it significantly. Apparently more care should have gone into cleaning up the deflector and the fringing field shunts. As conditioning progressed, it was seen that the electric strength of the gap was temporarily reduced by sparking. This slowed down the conditioning process and provided incentive to optimize the parameters to avoid sparks. The most significant tools to achieve this proved to be the stop limit and the hard limit. A stop limit of $5 \mu \mathrm{A}$, merely $1.5 \mu \mathrm{A}$ above the measured anode steady-state leakage current at $450 \mathrm{kV}$ accross the gap, reduced sparking effectively when used together with a slow ramp rate of $1 \mathrm{kV} / \mathrm{min}$. This change brought about longer times spent burning as well as the elevated radiation and worsened vacuum readings typically associated with field emission. It therefore stands to reason that more protrusions were slowly blunted by electron emission, not blasted away by arcing. Setting the hard limit between lowering and discharge limits allowed one to take advan- tage of the greater reaction speed associated with it. A low hard limit also allowed for some time to pass before a high voltage was again introduced to the electrode which had sparked. With $I_{\mathrm{H}}=30 \mu \mathrm{A}, V_{\text {neg }}=225 \mathrm{kV}$ this time was $900 \mathrm{~ms}$. Cascades of sparks were seen less often after this change, possibly indicating that the impurities released into the vacuum volume from the surfaces by the spark drift sufficiently far away, so that a conducting channel is rarely present by the time the high voltage reaches it's previous value.

The anode was conditioned to $225 \mathrm{kV}$ using the limits $I_{\mathrm{S}}=5 \mu \mathrm{A}, I_{\mathrm{L}}=20 \mu \mathrm{A}, I_{\mathrm{D}}=100 \mu \mathrm{A}$ and $I_{\mathrm{H}}=40 \mu \mathrm{A}$. The procedure was repeated for the cathode, after which the electrodes were conditioned symmetrically from $300 \mathrm{kV}$ to $450 \mathrm{kV}$ accross the gap. Deconditioning at a rate of $0.5 \mathrm{kV} / \mathrm{h}$ was observed at electrode voltages exceeding $200 \mathrm{kV}$. Conditioning rates as high as $4 \mathrm{kV} / \mathrm{h}$ were seen.

Tests with beam have shown the electic field to be stable enough for accurate separation of masses. Dumping the primary beam through the anode opening works as well, provided that vertical focus of the primary beam is sufficiently far downstream of the target. Subsequent conditioning to $450 \mathrm{kV}$ after two months without HV took three days with first discharges seen at $160 \mathrm{kV}$ accross the gap.

\section{Conclusion}

The electrostatic deflector of MARA separator was conditioned to $450 \mathrm{kV}$ using an automated control system. This translates to an electric field of $3.2 \mathrm{MV} / \mathrm{m}$ and a maximum electric rigidity of $13 \mathrm{MV}$. Deconditioning rate for the deflector was found to be $0.5 \mathrm{kV} / \mathrm{h}$. The bremsstrahlung background produced by a well conditioned deflector is negligible and the monitoring and logging system enables one to assess the need for reconditioning easily.

\section{References}

[1] J. Sarén, et al., Absolute transmission and separation properties of the gas-filled recoil separator RITU, Nucl. Instrum. Methods Phys. Res., Sect. A 654 (1) (2011) 508 - 521. doi:http://dx.doi.org/10.1016/j.nima.2011.06.068.

[2] J. Sarén, et al., The new vacuum-mode recoil separator MARA at JYFL, Nucl. Instrum. Methods Phys. Res., Sect. B 266 (19-20) (2008) 4196 - 4200. doi:http://dx.doi.org/10.1016/j.nimb.2008.05.027.

[3] V. Y. Ushakov, Insulation of High-Voltage Equipment, Springer, 2003.

[4] R. Latham, High Voltage Vacuum Insulation: Basic Concepts and Technological Practice, Elsevier, 1995.

[5] T. Yasuoka, et al., Electrode conditioning characteristics in vacuum based on discharge current, Proc. ISETS07.

[6] G. Münzenberg, et al., A high voltage deflection condenser operated under high vacuum conditions, Nucl. Instrum. Methods 166 (3) (1979) 391 - 395. doi:http://dx.doi.org/10.1016/0029-554X(79)90526-3.

[7] S. Morinobu, et al., Performance of and experiments with the recoil separator CARP at RCNP, Nucl. Instrum. Methods Phys. Res., Sect. B 70 (1992) 331-342. doi:10.1016/0168-583X(92)95949-R.

[8] C. N. Davids, Computer control of the fragment mass analyzer at ATLAS, Nucl. Instrum. Methods Phys. Res., Sect. A 345 (3) (1994) 528 - 533. doi:http://dx.doi.org/10.1016/0168-9002(94)90511-8. 